Supporting information for article:

\title{
Laser induced wettability gradient surface of aluminum matrix used for directional transportation and collection of underwater bubbles
}

ZhiXia Zheng ${ }^{1, *}$, Huan Yang ${ }^{2}$, Yiqing Cao ${ }^{1}$ and ZiYi Dai ${ }^{3}$

1 School of Mechanical \& Electrical Engineering, PuTian University, PuTian 351100, China; 1259900841@qq.com(Z. Zheng), caoyiqing1987@163.com(Y.Cao)

2 Sino-Geman College of Intelligent Manufacturing, Shenzhen Technology University, Shenzhen 518118, China; hycug@163.com(H.Yang)

3 Department of Electrical \& Computer Engineering, National University of Singapore, Singapore 117576; daiziyi2019@163.com（Z.Dai） 


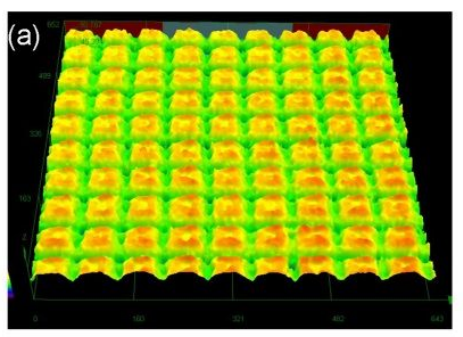

zone1

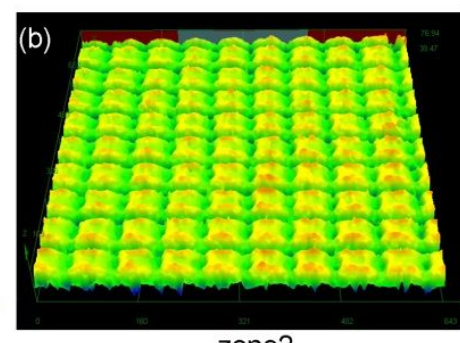

zone2

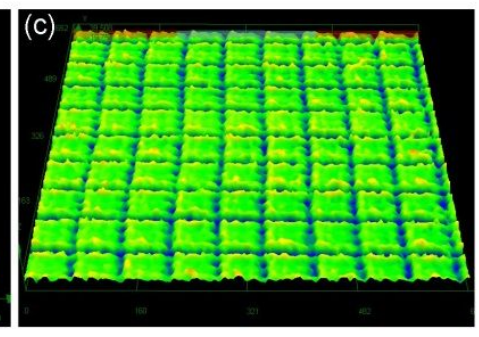

zone3

Figure S1. (a), (b) and (c) correspond to the laser scanning confocal electron microscope images of zone1, zone 2 and zone 3 , respectively. It shows that one end of the aluminum plate is raised, and the light spot is focused on the other end of the sample surface; and thus the surface with gradual roughness can be made.



Figure S2. SEM images of the laser textured sample surface. (a) Large-area view; (b) Further magnified image of near micropores; (c) Enlarged view of a microgroove; (d)Higher magnification image of micro/nano-particle aggregates. 